\title{
Echocardiographic Criteria of the Elderly
}

\section{Shaimaa A. Mostafa*}

Faculty of Medicine, Cardiovascular Department, Benha University, Egypt

*Corresponding author: Shaimaa A. Mostafa, Faculty of Medicine, Cardiovascular Department, Benha University, Egypt, Tel: 01229122843; E-mail: shaimaamustafa2011@gmail.com

Received date: February 17, 2015; Accepted date: March 20, 2015; Published date: March 24, 2015

Copyright: ( 2015 Mostafa SA. This is an open-access article distributed under the terms of the Creative Commons Attribution License, which permits unrestricted use, distribution, and reproduction in any medium, provided the original author and source are credited.

\section{Abstract}

Elderly are large sector of any community and they deserve our attention and care and this review article focus on the echocardiographic criteria in the elderly that may mimic or progress to cardiovascular disease.

Keywords: Echocardiography; Elderly

\section{Introduction}

\section{What is aging?}

Aging is the process of gradual and spontaneous changes resulting in maturation through childhood, puberty and young adulthood and then decline through middle and late age [1].

Like all visceral organs the heart ages. The fibrous skeleton of the heart becomes sclerotic and calcifies the valve closing margins, the aorta dilates and tilts rightward on the interventricular septum making the latter seem prominent, the ventricles decrease in size the left atrium enlarges, valves calcify and/or become myxomatous and amyloid may deposit in the heart [2].

Normal aging changes may produce clinical heart disease as sclerocalcific aortic valve progress to AS or the aging changes mimic heart disease as sigmoid septum and hypertrophic cardiomyopathy (Figure 1) [2].

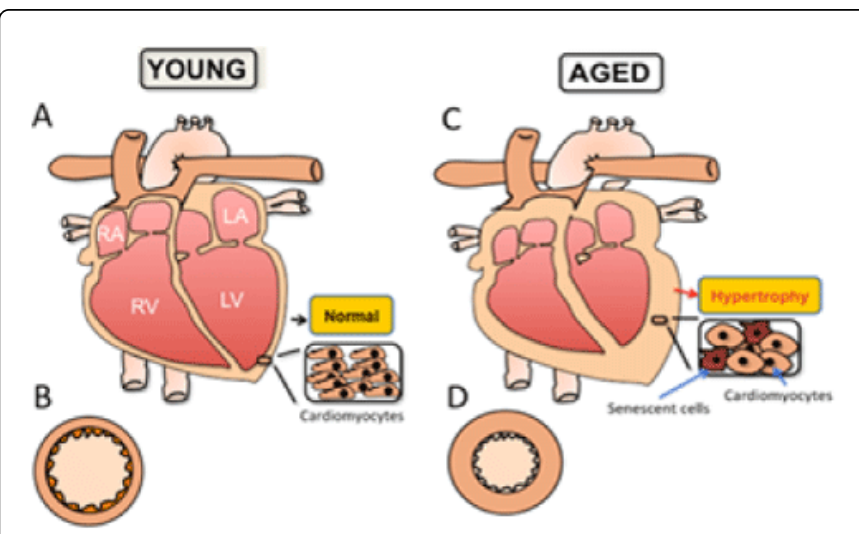

Figure 1: represents the cardiac and vascular changes in the elderly

Aged heart shows:

- Increased thickness in the heart muscle due to hypertrophy.

- Cardiomyocytes show hyperplasia
- Aged artery also has increased thickness, reduced lumen and less efficient contractile and relaxation properties

\section{Materials and Methods}

\section{Diastolic function in the elderly}

Aging is a physiological process accompanied by structural changes in both myocardium and arterial bed resulting in worsening left ventricular diastolic function as:

- Increased ventricular mass

- Collagen infiltration

- Altered myocardial calcium handling

This "physiological" diastolic dysfunction in the elderly can be explained by the deterioration of passive left ventricular filling properties and by worsening of left ventricular relaxation [3].

By TDI: $E^{\prime}$ at basal septal and basal lateral showed an inverse correlation with age (Figure 2). A velocity correlated directly with age, these manifestations may reflect a gradual decline in the left ventricular initial diastolic filling with a compensatory increase in the contribution of atrial systole to maintain an appropriate ventricular filling volume [3].

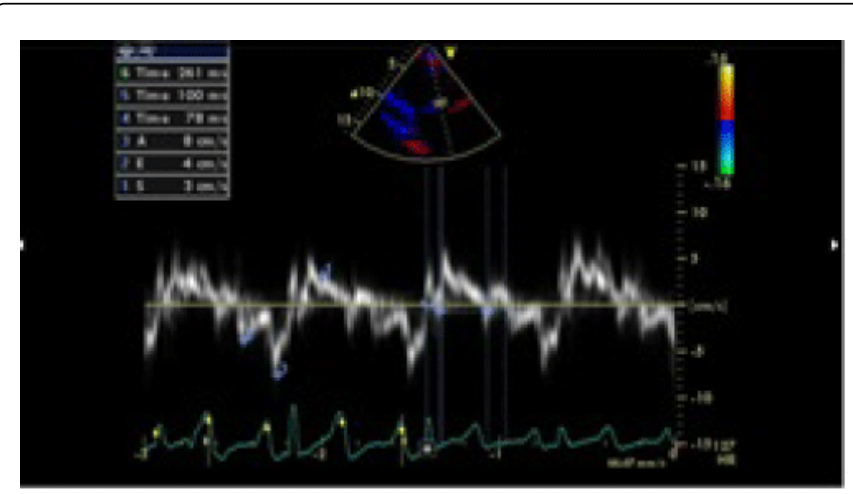

Figure 2: TDI of the basal septal segment

But the question is: How to distinguish the "physiological" diastolic dysfunction resulting from physiological tissue ageing from 
"pathological" diastolic dysfunction reflecting a disease of cardiovascular system?

\section{Systolic function in the elderly}

Resting left ventricular systolic function is not altered by aging in most studies, declines of stroke volume with sedentary older populations. Contractile responses to beta-adrenergic responses are decreased with aging [4].

\section{Valvular changes}

\section{1-Lambl's excrescences}

Lambl's excrescences and fibrous tags are thought to represent a common valvular degenerative change and often thought to reflect wear and tear (Figure 3). Some consider these to represent repetitive trauma related endothelial proliferations or organized thrombi [5].

Commonly occur at lines of valve closure and are most common on the left side, It may be single strand, in clusters, or raw, diameter about $1 \mathrm{~mm}$, and length 1-10 mm. Risk for ischemic stroke and transient ischemic attack [5].

The point is the differentiation between Lambl's excrescences and papillary fiberoelatoma which is difficult.

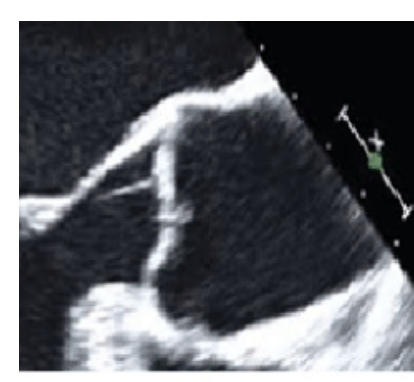

A

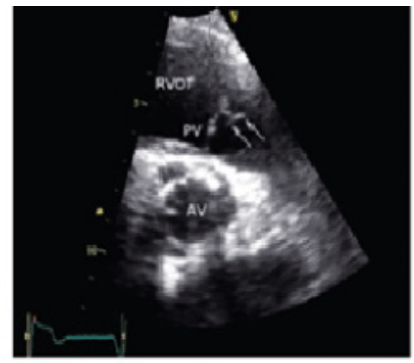

B
Figure 3: shows fibrous tags on the (A) aortic valve and (B) pulmonary valve

\section{2-Aortic valve changes}

Aortic valve changes thought to be passive in nature representing dystrophic calcification of degenerated material from wear and tear of the valve tissue. Followed by lipid accumulation, inflammation, alteration of cytokines, growth factors and valve MMPS. The process of valve calcification has much in common with atherosclerosis and bone formation (Figure 4). About $10 \%$ of individuals with aortic sclerosis will progress to develop aortic stenosis after 5yrs (Figure 5) [6].

\section{3-Mitral Annular Calcification}

Mitral annular calcification is usually localized to the mitral ring, invariably the most common site being the base of the posterior mitral leaflet. Rarely the calcium extends onto the mitral leaflet. This process generally starts at the base of the leaflets and the tips of leaflets remain mobile. Mitral annular calcification may be complicated by: MR, MS, $\mathrm{CHB}$ or embolic complications [7]. MAC can be distinguished from post-rheumatic changes by the lack of leaflet commissural fusion and the fact that the leaflet is not diffusely diseased (Figure 6).

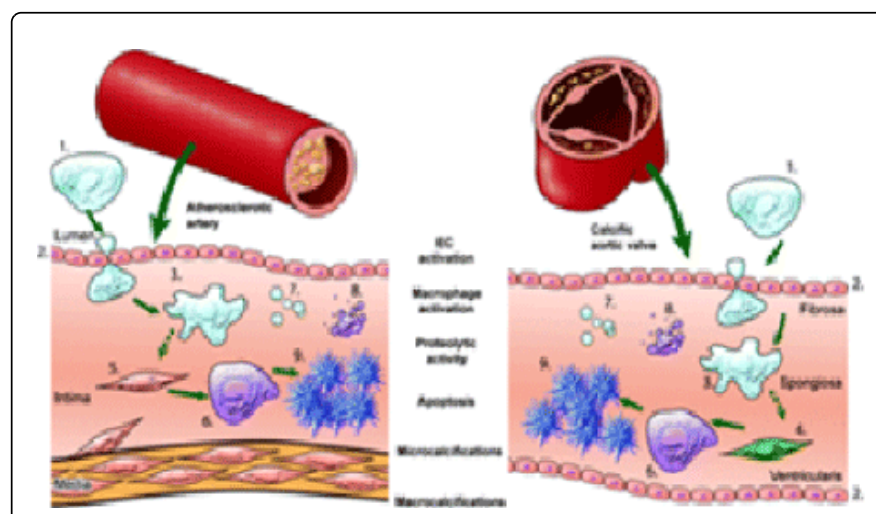

Figure 4: shows mechanism of atherosclerosis and aortic valve calcification

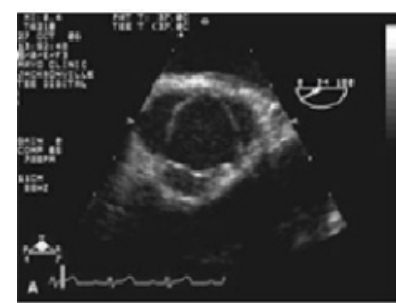

A

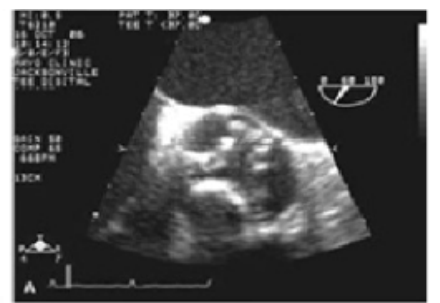

B
Figure 5: (A) normal tricuspid aortic valve and (B) sclerocalcific aortic valve

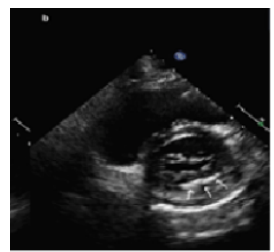

(a)

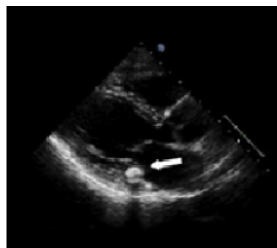

(b)

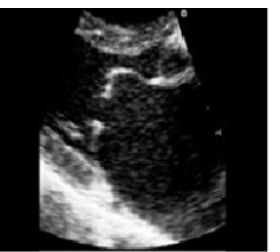

(c)
Figure 6: (a) mitral annulus calcification in the short axis view at the level of mitral valve and (b) parasternal long axis but (c) shows case of rheumatic mitral valve affection

\section{4-Mitral Valve Leaflet buckling}

As left ventricular cavity size decreases with advancing age, the area containing the mitral leaflets and chordae tendineae is reduced. Thus, during ventricular systole the mitral leaflet "buckle" or protrude into the left atrium. This leaflet protrusion may mimic the mitral valve prolapse pattern seen with a floppy valve.

Although floppy valves are occasionally observed in the elderly patient the vast majority of the valve protrusions is not the result of prolapsing floppy valves, but rather the consequence of changes in left ventricular cavity size. Anatomically, the elderly "buckling valve" has 
normal leaflet area and normal or reduced annular circumference in contrast to the increased leaflet area and dilated annular circumference of the floppy mitral valve. Mitral annular calcium in the elderly heart also may accentuate the leaflet buckling with mounds of calcium beneath the posterior leaflet.

Echocardiographic distinction of the elderly normal buckling mitral valve from the abnormal floppy mitral valve can be made by observing thickened and redundant leaflets of the floppy valve compared to thinly appearing leaflets of the normal old-age mitral valve (Figure 7). In addition, the degree of leaflet protrusion is generally greater in the floppy mitral valve than in the "buckling" old-age valve. A dilated left atrium will not help separate the floppy from the normal old-age mitral valve since left atrial dilation is present in both conditions [7].

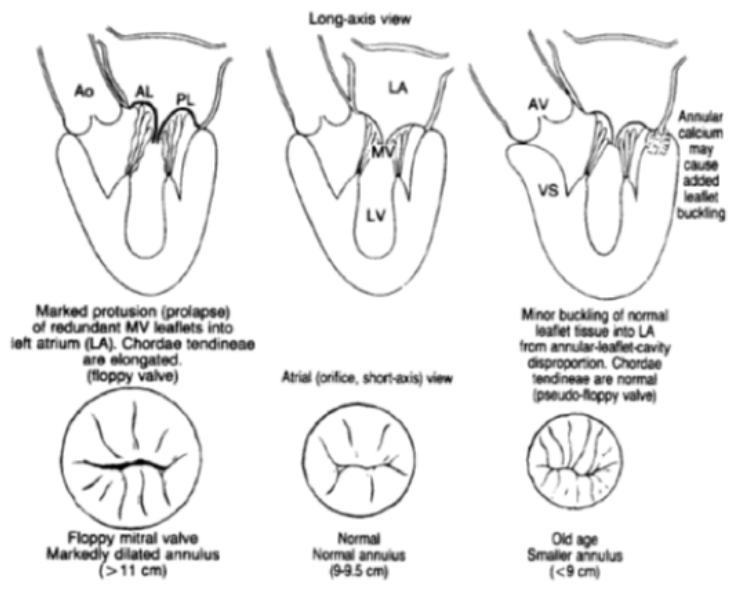

Figure 7: diagram representing the mitral valve apparatus in normal, floppy valve and old age

\section{Senile amyloidosis}

The heart may be involved by primary amyloid, secondary amyloid, and senile orange-related systemic and familial types. Amyloid may be clinically silent, or maybe associated with:

- arrhythmias

- diastolic dysfunction

- restrictive myocardial findings

- asymmetrical septal hypertrophy

- systolic dysfunction

- conduction disturbances

- coronary insufficiency

Echocardiographic finding in patients with amyloidosis:

- Ventricular wall thickening in the absence cavity dilatation.

- Ejection fraction is often normal.

- A decreased transmitral A wave can be due to the direct effect of atrial infiltration and normal $\mathrm{E}$ wave deceleration time with small A wave and can be encountered as refection of diastolic dysfunction.

- Pericardial effusion is common [8].

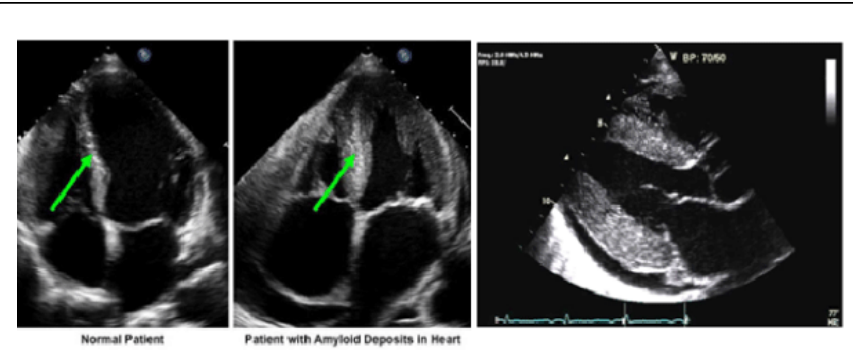

Figure 8: case of amyloid deposition in the heart
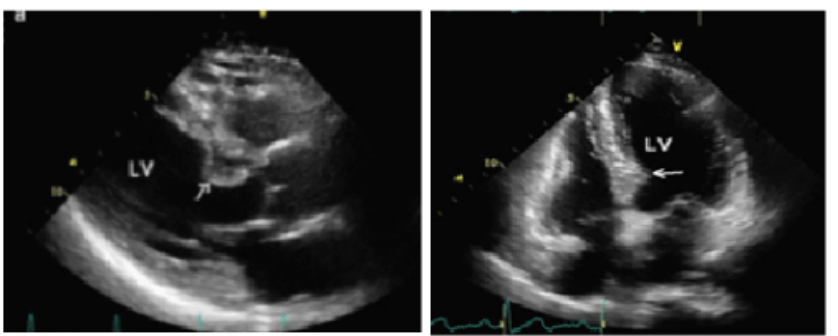

Figure 9: Bulging of the basal septal segment in the LVOT

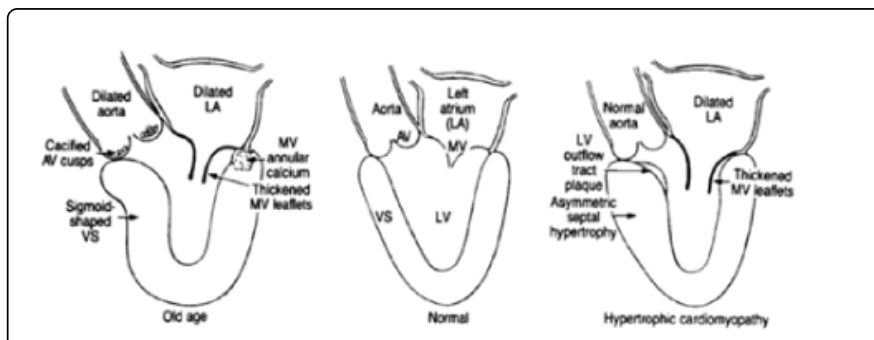

Figure 10: Shows the changes in hypertrophic cardiomyopathy and in old age resulting in sigmoid septum

\section{Sigmoid septum}

Sigmoid septum occurs partly as a result of the decrease in long-axis dimension of the LV and partly as a result of a rightward shift of the dilated ascending aorta so the basal ventricular septum bends leftward, bulging into the LVOT (Figure 9) [9].

Both the old-age heart and hypertrophic cardiomyopathy have: small LV cavities, thickened chamber walls, thickened mitral valve leaflets, and dilated LA cavities (Figure 10). In contrast the old-age heart has a dilated ascending aorta, thickened and calcified aortic valve cusps and mitral annular calcium [9].

\section{References}

1. Anversa P, Leri A, Kajstura J, Nadal-Ginard B (2002) Myocyte growth and cardiac repair. J Mol Cell Cardiol 34: 91-105.

2. Waller BF (1988) The old-age heart: normal aging changes which can produce or mimic cardiac disease. Clin Cardiol 11: 513-517. 
3. Márcia P, Iran C, Domingos H, José Carlos H, Flávia F (2004) Changes in the Parameters of Left Ventricular Diastolic Function According to Age on Tissue Doppler Imaging. Arquivos Brasileiros de Cardiologia 83.

4. Lakatta EG (2002) Introduction: chronic heart failure in older persons. Heart Fail Rev 7: 5-8.

5. Marina L, Vladimir T, Eli P, Ruthie S, Ricardo K, et al. (2014) Clinica significance and prevalence of valvular strands during routine echo examinations. European Heart Journal - Cardiovascular Imaging 15 1226-1230.

6. Mohler ER 3rd, Gannon F, Reynolds C, Zimmerman R, Keane MG, et al (2001) Bone formation and inflammation in cardiac valves. Circulation 103: $1522-1528$
7. Sahasakul Y, Edwards WD, Naessens JM, Tajik AJ (1988) Age related changes in aortic and mitral valve thickness: implications for twodimensional echocardiography based on an autopsy study of 200 normal human hearts. Am J Cardiol 62: 424-430.

8. Falk RH (2005) Diagnosis and management of the cardiac amyloidoses. Circulation 112: 2047-2060.

9. Ranasinghe I, Cheruvu C, Yiannikas J (2010) Sigmoid Septum (SS): An Age Related Phenomenon or Sigmoid Hypertrophic Cardiomyopathy (s HCM)? Heart and lung circulation 19: 53. 